\title{
INTELIGENCIA ARTIFICIAL Y AGRICULTURA: NUEVOS RETOS EN EL SECTOR AGRARIO ${ }^{1}$
}

\section{Francisca Ramón Fernández ${ }^{2}$}

\section{Resumen}

Objetivo del estudio: Analizar la aplicación de la inteligencia artificial a la agricultura, los distintos usos y legislación aplicable.

Metodología/enfoque: Se utiliza una metodología basada en el estudio de la legislación aplicable, así como el análisis de la doctrina sobre la materia. Sobre ello se formula una hipótesis que se basa en la deducción doctrinal para la aplicación a las cuestiones planteadas.

Originalidad/Relevancia: El presente estudio muestra la relación entre agricultura e inteligencia artificial con aplicación de la legislación más reciente, y con la aportación de soluciones a los distintos problemas planteados en relación al sector agrario que tiene como reto una informatización en su actividad.

Resultados principales: Se evidencian diversas aplicaciones de la inteligencia artificial en el ámbito de la agricultura, como es el caso de máquinas y sensores para diversas actividades agrarias. Distinta legislación aplicable a la misma. Diversas cuestiones en relación con los datos utilizados en las aplicaciones y su régimen de protección.

Contribuciones teóricas/metodológicas: Aplicación de la inteligencia artificial a la agricultura y necesidad de elaboración de legislación propia en la materia.

Contribuciones sociales/de gestión: Necesidad de establecer buenas prácticas en la gestión de la informática aplicada a la agricultura en relación con la inteligencia artificial.

Palabras clave: Inteligencia artificial. Agricultura. Robótica. Datos. Legislación.

\begin{tabular}{c}
\hline Recibido: 10/03/2020 \\
Aprobado: 09/05/2020 \\
Double Blind Review Process \\
DOI: $\underline{\text { https://doi.org/10.37497/revcampojur.v8i2.662 }}$ \\
\hline
\end{tabular}

\footnotetext{
${ }^{1}$ Trabajo rea lizado en el marco del Proyecto $\mathrm{I}+\mathrm{D}+\mathrm{i}$ "Retos de investiga ción" del Programa Esta talde I $+\mathrm{D}+\mathrm{i}$ orientada a los retos de la sociedad del Ministerio de Ciencia, Innovación y Universidades "Contratos, transparencia y protección de datos en el mercado digital” (2019-2022) (RTI2018-097354B-100). Investigadores Principales: Dr. D. Javier Plaza Penadés. Catedrático de Derecho civil y Dra. D ${ }^{\mathrm{a}}$. Luz M. Martínez Velencoso. Catedrática de Derecho civil. Universitat de València, y Proyecto de I+D $+\mathrm{i}$ "Retos de Investigación" del Programa Estatal de I+D+i orientada a los retos de la sociedad, MICINN "Derechos y garantías frente a las decisiones automatizadas en entornos de inteligencia artificial, IoT, big data y robótica" (PID2019-108710RB-I00, 2020-2022). Investiga dor principal: Dr. D. Lorenzo Cotino Hueso. Catedrático de Derecho Constitucional. Universitat de València.

2 Profesora titular de Derecho civil. Escuela Técnica Superior de Ingeniería del Diseño (ETSID). Universitat Politècnica de València,(España).E-mail: frarafer@urb.upv.es Orcid Id: http://orcid.org/0000-0002-0936$\underline{8229}$ Ficha UPV: http://www.upv.es/ficha-personal/frarafer
}

Rev. Campo Juridico, barreiras-BA v.8 n.2, p. 123-139, Julio-Diciembre, 2020. 


\title{
ARTIFICIAL INTELLIGENCE AND AGRICULTURE: NEW CHALLENGES IN THE AGRICULTURALSECTOR
}

\begin{abstract}
Objetive of the study: Analyze the application of artificial intelligence to agriculture, the different uses and applicable legislation.

Methodology/approach: Methodology based on the study of the applicable legislation is used, as well as the analysis of the doctrine on the matter. On this a hypothesis is formulated that is based on the doctrinal deduction for the application to the questions raised.
\end{abstract}

Originality/Relevance: This study shows the relationship between agriculture and artificial intelligence with the application of the most recent legislation, and with the contribution of solutions to the different problems posed in relation to the agricultural sector that has as a challenge computerization in its activity.

Main results: Various applications of artificial intelligence are evidenced in the field of agriculture, such as machines and sensors for various agricultural activities. Different legislation applicable to it. Various issues in relation to the data used in the applications and its protection regime.

Theoretical/methodological contributions: Application of artificial intelligence to agriculture and the need to draft own legislation on the matter.

Social/management contributions: Need to establish good practices in the management of informatics applied to agriculture in relation to artificial intelligence.

Keywords: Artificial intelligence. Farming. Robotics Data. Legislation. 


\title{
1 INTRODUCCIÓN
}

Las nuevas tecnologías de la información y comunicación (TICs) han producido una evolución en la sociedad actual tanto en el sector de servicios como en el sector de la producción. Tecnologías que se aplican al ámbito agrario influyendo en la explotación a graria y posterior comercialización de los productos agroalimentarios. Si nos referimos a la inteligencia artificial, robótica, el blockchain, los drones, la informática de alto rendimiento o la internet de las cosas, se pueden utilizar y de hecho se utilizan para incrementar la eficiencia de la agricultura, y en definitiva en potenciar la sostenibilidad y la seguridad y garantía de los productos.

No obstante, estas TICs se deben aplicar en sintonía con la legislación aplicable, ya que también nos referimos a los datos, a datos masivos, big data, y a responsabilidad, aspectos que no podemos dejar de lado cuando se utilizan herramientas basadas en la inteligencia artificial.

En este sentido, resulta de interés hacer referencia al concepto de macrodato y que la Resolución del Parlamento Europeo, de 14 de marzo de 2017, sobre las implicaciones de los macrodatos en los derechos fundamentales: privacidad, protección de datos, no discriminación, seguridad y aplicación de la ley $(2016 / 2225 \text { (INI) })^{3}$ y que lo concibe referido a:

\begin{abstract}
"la recopilación, análisis y acumulación constante de grandes cantidades de datos, incluidos datos personales, procedentes de diferentes fuentes y objeto de un tratamiento automatizado mediante algoritmos informáticos y avanzadas técnicas de tratamiento de datos, utilizando tanto datos almacenados como datos transmitidos en flujo continuo, con el fin de generar correlaciones, tendencias y patrones (analítica de macrodatos)".
\end{abstract}

Y que estos macrodatos se pueden utilizar tras la recogida de los mismos en dispositivos de inteligencia artificial para predecir acontecimientos, como por ejemplo, futuras inclemencias meteorológicas que puedan afectar a los cultivos, o bien plagas o alteraciones del suelo por contaminación.

Esta nueva revolución tecnológica, la revolución inteligente se aplica al campo, a la agricultura, siendo distintos los usos que nos podemos encontrar. El agricultor ya no va a limitarse a explotar sin servirse de las herramientas tecnológicas, sino que va a ir más allá.

3 Disponible en: < https://op.europa.eu/es/publication-detail//publication/bba9b363-8fdf-11e8-8bc101aa75ed71a1 >. Acceso en: 23 dic. 2020.

Rev. Campo Juridico, barreiras-BA v.8 n.2, p. 123-139, Julio-Diciembre, 2020. 
Desde la instalación de sensores en los árboles, a parametrar el terreno, programar el riego, determinar mediante un algoritmo la medida adecuada para fertilizar los cultivos, entre otros.

La modernización del sector agrícola pasa por su informatización, adaptando los aperos de labranza, y la maquinaria a la inteligencia artificial para poder controlar y aprovechar sin desperdicio el cultivo.

Ello, además, supone un incentivo para el agricultor joven que puede ver en la agricultura inteligente un medio de vida más avanzado que el de sus ancestros, y un rendimiento óptimo de la cosecha, sirviéndose de las TICs, en definitiva de algoritmos y métodos matemáticos de previsión del comportamiento de una actividad.

Así, por ejemplo, interesa destacar el Reglamento (UE) 2018/1807 del Parlamento Europeo y del Consejo, de 14 de noviembre de 2018, relativo a un marco para la libre circulación de datos no personales en la Unión Europea (Texto pertinente a efectos del EEE) ${ }^{4}$ en el que se expresa respecto a la agricultura que:

"Entre los ejemplos específicos de datos no personales se encuentran los conjuntos de datos agregados y anonimizados utilizados para análisis de datos a gran escala, los datos sobre agricultura de precisión que pueden ayudar a controlar y optimizar la utilización de plaguicidas y de agua, o los datos sobre las necesidades de mantenimiento de máquinas industriales. $\mathrm{Si}$ los avances tecnológicos hicieran posible transformar datos anónimos en datos personales, dichos datos se deben tratar como datos personales".

Por tanto, en el caso de que se transformaran en datos personales, sería de aplicación lo indicado en el Reglamento (UE) 2016/679 del Parlamento Europeo y del Consejo, de 27 de abril de 2016, relativo a la protección de las personas físicas en lo que respecta al tratamiento de datos personales y a la libre circulación de estos datos y por el que se deroga la Directiva 95/46/CE (Reglamento general de protección de datos) (Texto pertinente a efectos del EEE). ${ }^{5}$

Se trata del resultado final de la Propuesta de la Comisión Europea de 13 de septiembre de 2017 (COM/2017/0495 final) que concretó lo indicado en la Comunicación de la Comisión al Parlamento Europeo, al Consejo, al Comité Económico y Social Europeo y al Comité de las Regiones sobre "Una Estrategia para el Mercado Único Digital de Europa

\footnotetext{
${ }^{4}$ Disponible en: $<$ https://eur-lex.europa.eu/legal-content/es/ALL/?uri=CELEX\%3A32018R1807 >Acceso en: 22 dic. 2020.

5 Disponible en: < https://eur-lex.europa.eu/legal-content/ES/ALL/?uri=celex\%3A32016R0679> Acceso en: 23 dic. 2020.
}

Rev. Campo Juridico, barreiras-BA v.8 n.2, p. 123-139, Julio-Diciembre, 2020. 
(COM/2015/0192 final), en su apartado 4.1. ${ }^{6}$ (MASSENO, 2019; MASSENO, 2020) y estableció lo siguiente:

"La Comisión propondrá en 2016 una iniciativa europea de «libre circulación de datos» que aborde las restricciones a la libre circulación de los datos por motivos distintos de la protección de datos personales en la UE y las restricciones injustificadas a la ubicación de los datos para su almacenamiento o tratamiento. También se tratarán las cuestiones emergentes de propiedad, interoperabilidad, utilizabilidad y acceso a los datos en situaciones como datos de empresa a empresa, de empresa a consumidor, generados por una máquina y de máquina a máquina. Se fomentará el acceso a los datos públicos para ayudar a impulsar la innovación. La Comisión pondrá en marcha una iniciativa europea de computación en nube, incluida la certificación de servicios en nube, los contratos, cambio de proveedores de servicios en nube e investigación científica abierta en nube".

La Comunicación de la Comisión al Parlamento Europeo, al Consejo, al Comité Económico y social europeo y al Comité de las regiones relativa a la revisión intermedia de la aplicación de la Estrategia para el Mercado Único Digital Un mercado único digital conectado para todos (COM/2017/0228 final $)^{7}$ indica como ejemplo de datos no personales en relación con el ámbito agrario, los siguientes (Véase sobre ello: MASSENO, 2019; MASSENO, 2020):

"Como ejemplos de datos no personales cabe citar los registros fiscales, como facturas, documentos contables o documentos justificantes del registro de una empresa, los datos sobre agricultura de precisión (que ayudan a supervisar y optimizar el uso de plaguicidas, nutrientes y agua) o los proporcionados por sensores que comunican los datos que registran, como la temperatura o las condiciones del viento, por ejemplo en las turbinas eólicas, o los datos sobre las necesidades de mantenimiento de los robots industriales, por ejemplo cuando se quedan sin pintura”.

6 Disponible en: < https://eur-lex.europa.eu/legal-content/ES/TXT/?uri=CELEX\%3A52015DC0192 > Acceso en: 23 dic. 2020.

${ }^{7}$ Disponible en: $<$ https://eur-lex.europa.eu/legal-content/ES/TXT/?uri=COM\%3A2017\%3A228\%3AFIN $>$ Acceso en: 23 dic. 2020.

Rev. Campo Juridico, barreiras-BA v.8 n.2, p. 123-139, Julio-Diciembre, 2020. 


\section{LA INTELIGENCIA ARTIFICIAL EN EL ÁMBITO AGRARIO: ALGUNAS CONSIDERACIONES}

El Libro Blanco sobre la inteligencia artificial-un enfoque europeo orientado a la excelencia y la confianza ${ }^{8}$ señala que los avances en inteligencia artificial y sus sucesivas modificaciones con la evolución de la investigación mejorarán distintos ámbitos, como el sanitario y también aumentará la "eficiencia de la agricultura" con una mitigación del cambio climático, y su adaptación, además de mejorar "la eficiencia de los sistemas de producción a través de un mantenimiento predictivo.

Sin embargo, la inteligencia artificial también genera desconfianza entre la población en relación con la falta de transparencia de los algoritmos o el uso que se realicen de datos con fines no autorizados (RAMÓN, 2019, p. 1). De esta forma, hay que indicar la reciente iniciativa del Parlamento Europeo para aplicar directrices en relación a la inteligencia artificial (NOTICIAS PARLAMENTO EUROPEO, 2020), entre la que destaca la Resolución del Parlamento Europeo, de 20 de octubre de 2020, con recomendaciones destinadas a la Comisión sobre un régimen de responsabilidad civil en materia de inteligencia artificial $\left(2020 / 2014\left(\right.\right.$ INL)). ${ }^{9}$

La define el Libro Blanco a la inteligencia artificial como "una combinación de tecnologías que agrupa datos, algoritmos y capacidad informática”, ya que aúna computación, disponibilidad de los datos, y un gran potencial tecnológico e industrial para desarrollar la innovación y su aplicación.

La Comunicación de la Comisión al Parlamento Europeo, al Consejo, al Comité Económico y social europeo y al Comité de las Regiones sobre una Estrategia Europea de Datos (COM/2020/66 final $)^{10}$ menciona la importancia de los datos y su naturaleza esencial en distintos sectores que avanzan a velocidad diferente, siendo uno de los ámbitos estratégicos la agricultura.

Este documento ofrece información sobre el Espacio común europeo de datos relativos al sector agrario y se indicó que los datos forman parte de la información necesaria para mejorar los resultados respecto a la sostenibilidad y competitividad del sector agrario.

No sólo los datos referentes a la producción, sino también los relativos a la cadena de suministro, observación terrestre y meteorología. Estos datos proporcionan información

\footnotetext{
8 Disponible en: $<$ LIBRO BLANCO sobre la inteligencia artificial - un enfoque europeo orientado a la excelencia y la confianza COM/2020/65 fina1/2> Acceso en: 22 dic. 2020.

${ }^{9}$ Disponible en: $<$ https://www.europarl.europa.eu/doceo/document/TA-9-2020-0276 ES.pdf $>$ Acceso en: 23 dic. 2020.

${ }^{10}$ Disponible en: <https://eur-lex.europa.eu/legal-content/ES/TXT/?uri=CELEX:52020DC0066> Acceso en: 22 dic. 2020.
}

Rev. Campo Juridico, barreiras-BA v.8 n.2, p. 123-139, Julio-Diciembre, 2020. 
relevante para la explotación agraria, pudiendo predecir los fenómenos atmosféricos y la toma de medidas y precauciones para evitar daños en las cosechas. Se trata de predecir y precisar la explotación agraria basada en datos y fórmulas matemáticas.

Durante el año 2018 se elaboró por parte de la Unión Europea el Código de conducta para el intercambio de datos agrarios que se refería a los cultivos y la maquinaria. Como propósito se indica la creación de:

"Un espacio común de datos agrarios basado en los enfoques existentes para el intercambio de datos podría conducir a una plataforma neutral para intercambiar y agrupar datos agrarios, tanto públicos como privados. Esto podría contribuir a la aparición de un ecosistema innovador basado en datos y sustentado en relaciones contractuales justas, así como reforzar las capacidades para el seguimiento y la ejecución de las políticas comunes y reducir la carga administrativa para el Gobiemo y los beneficiarios".

La declaración de cooperación "Un futuro digital inteligente y sostenible para la agricultura y las zonas rurales europeas", de 2019, reconoció el potencial de las tecnologías digitales en la agricultura y la creación de espacios de datos.

En España destacar la aprobación e implantación de la Estrategia de digitalización del sector agroalimentario y forestal y del medio natural del Ministerio de Agricultura, Pesca y Alimentación ${ }^{11}$ mediante la cual se definen las líneas y medidas para la transformación digital de los sectores implicados.

Su objetivo, tal y como indica el documento, es "buscar la eliminación o reducción de las barreras técnicas, legislativas, económicas y formativas existentes en la actualidad, contribuyendo así al liderazgo de un sector agroalimentario sostenible económica, social y medioambientalmente, y al poblamiento activo del medio rural haciéndolo un lugar más atractivo, vivo, dinámico y diversificado, generador de riqueza y de empleo de calidad, con especial atención a jóvenes y mujeres”.

Para ello, se establecen tres objetivos bien diferenciados:

a) Reducción de la brecha digital fomentando la conectividad;

b) Fomento del uso de los datos con abordaje de la interoperabilidad de los datos y la apertura de los mismos, con apertura de los datos en diversos ámbitos: administrativo, investigación y sector privado;

11 Disponible en: < https://www.mapa.gob.es/es/ministerio/planes-estrategias/estrategia-digitalizacionsector-agroalimentario/estrategia_digitalizacion_sector_agroalimentario_forestal_medio_rural_ve_tcm30509645.pdf > Acceso en: 23 dic. 2020.

Rev. Campo Juridico, barreiras-BA v.8 n.2, p. 123-139, Julio-Diciembre, 2020. 
c) Impulso del desarrollo de la empresa y creación de nuevos modelos de negocios en relación con la industria 4.0. Todo ello pasa por la modernización, asesoramiento digital del sector agroalimentario y forestal.

El I Plan de acción 2019-2020 Estrategia de digitalización del sector agroalimentario y forestal y del medio rural ${ }^{12}$ del Ministerio de Agricultura, Pesca y Alimentación de España recoge las actuaciones para desarrollar durante el periodo 2019-2020 para implementar la Estrategia de Digitalización del sector agroalimentario y forestal y del medio natural.

\section{ROBOTS AGRICULTORES}

Así, por ejemplo, entre los distintos proyectos financiados por la Unión Europea, destacan los siguientes (COMISIÓN EUROPEA, 2019) ${ }^{13}$ en los que destacan la creación de robots y la utilización de la inteligencia artificial para la recolección de productos del campo:

a) Sweeper. Se trata de un robot que recolecta pimientos dulces y que desarrolla la actividad en invernaderos. ${ }^{14}$

b) Data Driven Dairy Decision for Farmers (4D4F). Constituye una red para productores de leche, tecnología láctea, empresarios, médicos veterinarios y personal de investigación para la utilización de sensores con la finalidad de obtener datos para mejorar las granjas lecheras en función de la información proporcionada. ${ }^{15}$

c) Internet of Food \& Farm 2020. (IoF2020). Se refiere a un proyecto subvencionado con fondos de la Unión Europea para hacer realidad la agricultura de precisión. ${ }^{16}$

d) Data-Driven Bioeconomy (DataBio). Se trata de un proyecto lighthouse H2020 que utiliza big data para contribuir a unas mejores materias primas en el ámbito de la agricultura, forestal, acuicultura y pesca en las industrias de la bioeconomía para producción de alimentos, energía y biomateriales que sean sostenibles y responsables. ${ }^{17}$

e) SmartAgriHubs. Se concibe como un proyecto de la Unión Europea H2020 con la finalidad de digitalizar la agricultura europea a través de un ecosistema de innovación agrícola dedicado a la excelencia, sostenibilidad y éxito. ${ }^{18}$

12 Disponible en: < https://www.mapa.gob.es/es/ministerio/planes-estrategias/estrategia-digitalizacionsector-a groalimentario/plan-accion-2019-2020-estrategia-digitalizacion tcm30-524904.pdf $>$ Acceso en: 23 dic. 2020.

13 Disponible en: < https://ec.europa.eu/digital-single-market/en/news/eu-member-states-join-forcesdigitalisation-european-agriculture-and-rural-areas $>$ Acceso en: 22 dic. 2020.

${ }_{14}^{14}$ Disponible en: $<\mathrm{http}: / /$ www.sweeper-robot.eu/> Acceso en: 22 dic. 2020.

${ }^{15}$ Disponible en: $<$ https://www.4d4f.eu/> Acceso en: 22 dic. 2020.

${ }^{16}$ Disponible en: $<$ Internet of Food and Farm 2020 - IoF2020 $>$ Acceso en: 23 dic. 2020.

17 Disponible en: $<$ Databio $>$ Acceso en: 23 dic. 2020.

${ }^{18}$ Disponible en: < SmartAgriHubs | Homepage $>$ Acceso en: 23 dic. 2020.

Rev. Campo Juridico, barreiras-BA v.8 n.2, p. 123-139, Julio-Diciembre, 2020. 
f) Humane AI. Red Europea de Inteligencia Artificial centrada en el ser humano. La finalidad es facilitar una marca europea de IA ética que mejore las capacidades humanas y capacitar a los ciudadanos y la sociedad para hacer frente a los desafíos del mundo globalizado interconectado. ${ }^{19}$

g) Pantheon. Son robots que se han diseñado para moverse en el suelo y el aire para recoger avellana, además de recoger datos sobre los árboles, niveles del agua, y plagas o enfermedades de los ejemplares. ${ }^{20}$

h) Flourish. Robots diseñados para la agricultura de precisión, y que desarrollan actividades en la agricultura recopilando datos referentes al suelo, cultivos, plagas, agua, para incrementar el rendimiento de la explotación y minimizar el uso de productos químicos. ${ }^{21}$

Ello se pone en realización con la trazabilidad alimentaria (MESA y RAMÓN, 2016, p. 120) y la creación de aplicaciones agroalimentarias inteligentes, además de contribuir a la sostenibilidad y a los Objetivos de Desarrollo Sostenible (RAMÓN, 2020, p. 153), tal y como ha indicado la Resolución del Parlamento Europeo, de 20 de octubre de 2020, con recomendaciones destinadas a la Comisión sobre un marco de los aspectos éticos de la inteligencia artificial, la robótica y las tecnologías conexas (2020/2012 (INL)). ${ }^{22}$

\section{LA APLICACIÓN DE LOS DRONES EN LA AGRICULTURA}

Los denominados drones o aeronaves no tripuladas pilotadas por control remoto, también conocidas por las siglas RPAS (Remotely Piloted Aircraft Systems) han planteado distintas cuestiones en diversas áreas del Derecho. También se las ha denominado como aeronaves sin piloto vehículos aéreos no tripulados o UAVs (Unmanned Aerial Vehicle). Se incluyen tanto las aeronaves que realizan un vuelo sin ser pilotadas (bien por control remoto) o bien estando programadas y funcionar de forma autónoma.

Desde su propia denominación hasta su tratamiento y régimen jurídico que ha estado regulado de forma muy incompleta, teniendo en cuenta que se desplazan por el espacio aéreo, con lo que, junto con su coste cada vez más accesible, han ido proliferando de forma alarmante, y suponiendo un riesgo para la seguridad aérea.

\footnotetext{
${ }^{19}$ Disponible en: $<$ Humane AI $\mid$ Human-Centered Artificial Intelligence (humane-ai.eu) $>$ Acceso en: 23 dic. 2020.

${ }^{20}$ Disponible en: $<$ Pantheon: robots giving a helping hand to hazelnut farmers $\mid$ Shaping Europe's digital future (europa.eu) $>$ Acceso en: 23 dic. 2020.

${ }^{21}$ Disponible en: $<$ Flourish: robots for more productive and sustainable agriculture Shaping Europe's digital future (europa.eu) $>$ Acceso en: 23 dic. 2020.

${ }^{22}$ Disponible en: <https://www.europarl.europa.eu/doceo/document/TA-9-2020-0275_ES.pdf $>$ Acceso en: 23 dic. 2020.
}

Rev. Campo Juridico, barreiras-BA v.8 n.2, p. 123-139, Julio-Diciembre, 2020. 
El riesgo ya se indicaba en el Convenio sobre Aviación Civil Internacional de 7 de diciembre de 1944 conocido como Convenio de Chicago ${ }^{23}$, en su art. 8 , referente a aeronaves sin piloto, indica que:

"Ninguna aeronave capaz de volar sin piloto volará sin él sobre el territorio de un Estado contratante, a menos que se cuente con autorización especial de tal Estado y de conformidad con los términos de dicha autorización. Cada Estado contratante se compromete a asegurar que los vuelos de tales aeronaves sin piloto en las regiones abiertas a la navegación de las aeronaves civiles sean controlados de forma que se evite todo peligro a las aeronaves civiles".

Actualmente, en España se regulan por el Real Decreto 1036/2017, de 15 de diciembre, por el que se regula la utilización civil de las aeronaves pilotadas por control remoto, y se modifican el Real Decreto 552/2014, de 27 de junio, por el que se desarrolla el Reglamento del aire y disposiciones operativas comunes para los servicios y procedimientos de navegación aérea y el Real Decreto 57/2002, de 18 de enero, por el que se aprueba el Reglamento de Circulación Aérea $^{24}$, modificado por Real Decreto 1088/2020, de 9 de diciembre, por el que se completa el régimen aplicable a la notificación de sucesos de la aviación civil y se modifica el Real Decreto 1036/2017, de 15 de diciembre, por el que se regula la utilización civil de las aeronaves pilotadas por control remoto, y se modifican el Real Decreto 552/2014, de 27 de junio, por el que se desarrolla el Reglamento del aire y disposiciones operativas comunes para los servicios y procedimientos de navegación aérea y el Real Decreto 57/2002, de 18 de enero, por el que se aprueba el Reglamento de Circulación Aérea. ${ }^{25}$ Junto a ello, también se aplica el Real Decreto 1180/2018, de 21 de septiembre, por el que se desarrolla el Reglamento del aire y disposiciones operativas comunes para los servicios y procedimientos de nave gación aérea y se modifican el Real Decreto 57/2002, de 18 de enero, por el que se aprueba el Reglamento de Circulación Aérea; el Real Decreto 862/2009, de 14 de mayo, por el que se aprueban las normas técnicas de diseño y operación de aeródromos de uso público; el Real Decreto 931/2010, de 23 de julio, por el que se regula el procedimiento de certificación de proveedores civiles de servicios de navegación aérea y su control normativo; y el Reglamento de la Circulación Aérea Operativa, aprobado por Real Decreto 601/2016, de 2 de diciembre, ${ }^{26}$ y cuyo artículo 44 regula

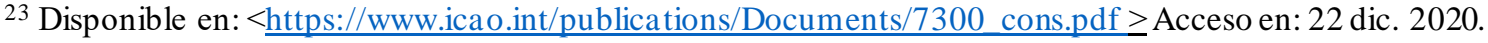

${ }^{24}$ BOE n. 316, de 29 de diciembre de 2017. Disponible en: $<$ https://www.boe.es/buscar/doc.php?id=BOEA-2017-15721 > Acceso en: 23 dic. 2020.

${ }^{25}$ BOE n. 322, de 10 de diciembre de 2020. Disponible en: $<$ https://www.boe.es/buscar/doc.php?id=BOEA-2020-15874 > Acceso en: 23 dic. 2020.

${ }^{26}$ BOE n. 272, de 10 de noviembre de 2018. Disponible en: $<$ https://www.boe.es/buscar/doc.php?id=BOEA-2018-15406 > Acceso en: 23 dic. 2020.
} 
las reglas del aire aplicables a las aeronaves pilotadas por control remoto que se aplicarán a las que no estén destinadas exclusivamente a actividades deportivas, recreativas, de competición y exhibición, así como a las actividades lúdicas, siendo de aplicación también lo indicado en el mencionado Real Decreto 1036/2017.

Ello ha motivado que los drones que inicialmente se utilizaran para usos recreativos, se hayan posicionado como un instrumento o herramienta en aras de una mejora de distintos espacios, como puede ser el agrario. La aplicación de los drones en el ámbito de la agricultura tiene diversos usos y funciones que la doctrina ha puesto de manifiesto, tanto en el caso de la teledetección aerotransportada en la agricultura de precisión (BARBA y SANCHIS, 2005, p. 40), como también para incrementar la eficiencia de los cultiv os (PINO, 2019, p. 75), y para la gestión de regadíos (VÁZQUEZ, RUIZ, MOLINA. y FERNÁNDEZ, 2012, p. 430).

Es lo que se denomina “agricultura inteligente” (DÍEZ, 2017,p. 4; TRIBALDOS, 2018, p. 60), ya que muchos dispositivos que se utilizan contienen algoritmos y son programados con la finalidad de servir a una mejora del ámbito agrario.

La agricultura de precisión es definida por la Resolución del Parlamento Europeo, de 7 de junio de 2016, sobre soluciones tecnológicas para una agricultura sostenible en la Unión $(2015 / 2225 \text { (INI) })^{27}$ como la agricultura que utiliza la "automatización y otras tecnologías para mejorar la precisión y la eficacia de prácticas clave de gestión agrícola y que se basa en planteamientos sistémicos para recopilar y analizar datos y optimizar las interacciones entre el clima, el suelo, el agua y los cultivos". Se concibe este tipo de agricultura para la reducción del uso de plaguicidas, abonos y agua, así como para mejorar la fertilidad del suelo y optimizar el rendimiento del campo.

Y se refiere a ella estableciendo lo siguiente:

“1. Señala que el sector agrícola siempre ha recurrido a nuevos modelos empresariales y prácticas agrícolas, incluidas nuevas técnicas y métodos de producción para aumentar el rendimiento y adaptarse a circunstancias nuevas y cambiantes; hace hincapié en que los servicios ecosistémicos, como los ciclos de nutrientes, son un elemento central de la agricultura y que algunas funciones, como la captura de carbono, van más allá de la producción de alimentos;

2. Está convencido de que la innovación alberga potencial para contribuir a lograr una agricultura sostenible en la Unión y considera que las tecnologías agrícolas de precisión

\begin{tabular}{llll}
\hline 27 & Disponible & en: & $<$ https://eur-lex.europa.eu/legal \\
content/ES/TXT/?uri=CELEX\%3A52016IP0251\&qid=1608742056010 & $>$ Acceso en:23 dic. 2020.
\end{tabular}

Rev. Campo Juridico, barreiras-BA v.8 n.2, p. 123-139, Julio-Diciembre, 2020. 
son especialmente importantes para seguir avanzando, pero reconoce las limitaciones que impiden su adopción de manera generalizada, en particular su fiabilidad, la capacidad para gestionarlas y los conocimientos limitados sobre estas tecnologías y la posibilidad de adaptarlas a explotaciones agrícolas de distinto tipo y tamaño;

3. Considera que los principios en los que se basa la agricultura de precisión pueden generar beneficios ambientales considerables, aumentar los beneficios de los agricultores, racionalizar la utilización de la maquinaria agrícola y mejorar significativamente la eficiencia en el uso de los recursos, incluido el uso del agua con fines de riego; anima, por tanto, a la Comisión a que promueva políticas que estimulen el desarrollo y la implantación de las tecnologías agrícolas de precisión para todos los tipos de explotación, con independencia de su tamaño y de su producción, tanto para explotaciones agrícolas como ganaderas;

4. Destaca la necesidad concreta de que el proceso de innovación asociado a la agricultura de precisión resuelva el problema del «alto coste» que suponen el desarrollo y el uso de algunas tecnologías de dicha agricultura, y de que toda la cadena de suministro participe activamente en el desarrollo de esas tecnologías para garantizar que las explotaciones obtengan beneficios indiscutibles y contribuir a que sean más resilientes;

5. Expresa su convencimiento de que el desarrollo económico y la producción sostenible no se excluyen mutuamente y pueden alcanzarse a través de la innovación; destaca la necesidad de apoyar la innovación en materia de tecnología y gobernanza, garantizando que existen coherencia y claridad normativas y oportunidades para emprender, e insta a la Comisión a que garantice que en próximas revisiones y reformas de la legislación pertinente se tenga explícitamente en cuenta la innovación; destaca que la agricultura europea es capaz de producir productos de una calidad y un valor añadido elevados, así como de ofrecer soluciones rentables basadas en el conocimiento para alimentar a una población mundial cada vez más numerosa y exigente;

6. Pide a la industria, a la Comisión y a los Estados miembros que trabajen conjuntamente para mejorar el rendimiento y la adaptabilidad de la robóticay otras técnicas empleadas en la agricultura de precisión a fin de que los fondos asignados a la investigación se utilicen de manera efectiva en el interés de la agricultura y la horticultura;

7. Pide al sector que aproveche las oportunidades que ofrece la innovación para desarrollar las capacidades de la agricultura de precisión que están a disposición de todo el mundo, empoderando de ese modo a las personas con discapacidad, promoviendo la igualdad de género y ampliando la base de competencias y las oportunidades de empleo en las comunidades rurales;

8. Acoge con satisfacción la inclusión de la robótica aplicada a la agricultura de precisión en el programa de trabajo de Horizonte 2020 para el período 2016-2017, recientemente 
publicado, perolamenta que no se exija que las propuestas presentadas en el marco de dicha convocatoria adopten un enfoque multilateral, lo que puede tener como consecuencia la exclusión de los agricultores de los enfoques innovadores; hace hincapié en que la agricultura de precisión puede reducir el consumo de recursos en al menos un $15 \%$; alienta el uso de la agricultura de precisión, que ofrece nuevos enfoques de gestión integral de las explotaciones, como la maquinaria dotada de tecnología GPS/GNSS y los sistemas de aeronaves pilotadas a distancia (RPAS)".

Alude también a la Agencia Espacial Europea (ESA) en relación al satélite Sentinel 2B y que proporcionará una visión más precisa de la cubierta de los cultivos y de la densidad forestal con la finalidad de racionalizar los recursos y periodos más adecuados para la cosecha.

\section{CONCLUSIONES}

La digitalización de la agricultura poco a poco es una realidad. La inteligencia artificial aplicada al sector agroalimentario of rece nuevas posibilidades de mejora de la explotación y de la sostenibilidad de los recursos.

Son muchas las interrogantes que se formulan en torno a las herramientas e instrumentos para informatizar la agricultura, a través de la implantación de sensores, y la utilización de algoritmos para predecir el comportamiento y la obtención de datos con la finalidad de aplicarlos en los cultivos y la ganadería, y posteriormente también en la fase de comercialización del producto. Sin olvidar el destinatario final, el consumidor, que también a través de la inteligencia artificial, en las denominadas ciudades inteligentes, "Smart Cities", puede mostrar su orientación hacia la compra de un productoo servicio. Las denominadas granjas inteligentes "Smart Farming" o campos inteligentes "Smart Fields" también pretenden optimizar sus recursos (a través de sistemas de alimentación o de riego mediante software) y facilitar un producto que sea consumible y sostenible al mismo tiempo.

La necesidad de una legislación específica es una demanda que debe ser atendida, ya que son muchas las lagunas que se pueden plantear en dicho ámbito, desde la responsabilidad civil por daños hasta la protección de los datos en relación con la Ley Orgánica 3/2018, de 5 de diciembre, de Protección de Datos Personales y garantía de los derechos digitales. ${ }^{28}$

Junto a ello, la normativa actual respecto de las aeronaves no tripuladas pilotadas por control remoto, también conocidas por las siglas RPAS, o drones, establecen una serie de consideraciones para la utilización de las mismas para usos no lúdicos, entre los que se encuentra la utilización en los cultivos, así como también el uso de satélites que orbitan la tierra y que proporcionan información sobre los cultivos y densidad de los bosques.

${ }^{28}$ BOE n. 294, de 6 de diciembre de 2018. Disponible en: < https://www.boe.es/buscar/doc.php?id=BOEA-2018-16673 > Acceso en: 23 dic. 2020.

Rev. Campo Juridico, barreiras-BA v.8 n.2, p. 123-139, Julio-Diciembre, 2020. 


\section{REFERENCIAS}

BARBA POLO, Juan y SANCHIS MUÑOZ, Javier. "Teledetección aerotransportada: caso de estudio de la agricultura de precisión". Mapping, Ejemplar dedicado a Jornada sobre normativa, pilotaje y aplicaciones de aeronaves no tripuladas (drones), n. 171, 2005, pág. 40 -43.

Data-Driven Bioeconomy (DataBio). Disponible en: $<$ Databio $>$ Acceso en: 23 dic. 2020.

Data Driven Dairy Decision for Farmers (4D4F). Disponible en: $<$ https://www.4d4f.eu/ $>$ Acceso en: 22 dic. 2020.

DÍEZ, Charo. "Hacia una agricultura inteligente”. Cuadernos del campo, n. 60, 2017, pág. 4-11. Disponible en: <http://www.larioja.org/lariojaclient/cm/agricultura/images?idMmedia=930866> Acceso en: 22 dic. 2020.

ESPAÑA. Real Decreto 1036/2017, de 15 de diciembre, por el que se regula la utilización civil de las aeronaves pilotadas por control remoto, y se modifican el Real Decreto 552/2014, de 27 de junio, por el que se desarrolla el Reglamento del aire y disposiciones operativas comunes para los servicios y procedimientos de navegación aérea y el Real Decreto 57/2002, de 18 de enero, por el que se aprueba el Reglamento de Circulación Aérea. BOE n. 316, de 29 de diciembre de 2017). Disponible en: $<$ https://www.boe.es/buscar/doc.php?id=BOE-A-2017-15721 >Acceso en: 23 dic. 2020.

ESPAÑA. Real Decreto 1180/2018, de 21 de septiembre, por el que se desarrolla el Reglamento del aire y disposiciones operativas comunes para los servicios y proc edimientos de navegación aérea y se modifican el Real Decreto 57/2002, de 18 de enero, por el que se aprueba el Reglamento de Circulación Aérea; el Real Decreto 862/2009, de 14 de mayo, por el que se aprueban las normas técnicas de diseño y operación de aeródromos de uso público; el Real Decreto 931/2010, de 23 de julio, por el que se regula el procedimiento de certificación de proveedores civiles de servicios de navegación aérea y su control normativo; y el Reglamento de la Circulación Aérea Operativa, aprobado por Real Decreto 601/2016, de 2 de diciembre. BOE $n$. 272, de 10 de noviembre de 2018. Disponible en: $<$ https://www.boe.es/buscar/doc.php?id=BOEA-2018-15406 > Acceso en: 23 dic. 2020.

ESPAÑA. Ley Orgánica 3/2018, de 5 de diciembre, de Protección de Datos Personales y garantía de los derechos digitales. BOE n. 294, de 6 de diciembre de 2018. Disponible en: < https://www.boe.es/buscar/doc.php?id=BOE-A-2018-16673 > Acceso en: 23 dic. 2020.

ESPAÑA. Real Decreto 1088/2020, de 9 de diciembre, por el que se completa el régimen aplicable a la notificación de sucesos de la aviación civil y se modificael RealDecreto 1036/2017, de 15 de diciembre, por el que se regula la utilización civil de las aeronaves pilotadas por control remoto, y se modifican el Real Decreto 552/2014, de 27 de junio, por el que se desarrolla el Reglamento del aire y disposiciones operativas comunes para los servicios y procedimientos de navegación aérea y el Real Decreto 57/2002, de 18 de enero, por el que se aprueba el Reglamento de Circulación Aérea. BOE n. 322, de 10 de diciembre de 2020. Disponible en: < https://www.boe.es/buscar/doc.php?id=BOE-A-2020-15874 > Acceso en: 23 dic. 2020.

Flourish. Disponible en: $<$ Flourish: robots for more productive and sustainable agriculture Shaping Europe's digital future (europa.eu) > Acceso en: 23 dic. 2020.

Humane AI. Red Europea de Inteligencia Artificial centrada en el ser humano. Disponible en: $<$ Humane AI $\mid$ Human-Centered Artificial Intelligence (humane-ai.eu) $>$ Acceso en: 23 dic. 2020 . 
Internet of Food \& Farm 2020. (IoF2020). Disponibleen: $<$ Internet of Food and Farm 2020 - IoF2020> Acceso en: 23 dic. 2020.

MASSENO, Manuel David. "Los datos no personales en las nuevas reglas europeas y su relevancia para los agricultores-Una guía para el estudio". Campo jurídico: Revista de direito agroambiental e teoria do direito, vol. 7, n. 2, pág. 122-144. Disponible en: $<$ http://www.fasb.edu.br/revista/index.php/campojuridico/article/view/549/455> Acceso en: 23 dic. 2020 .

MASSENO, Manuel David. "Los datos no personales en las nuevas reglas europeas y su relevancia para los agricultores-Una guía para el estudio". Marco Jurídico de la Ciencia de Datos, RAMÓN FERNÁNDEZ, Francisca (Coord.), Tirant lo Blanch, Valencia, 2020, pág. 301-329.

MESA GARCÍA, Olaya. y RAMÓN FERNÁNDEZ, Francisca. "La trazabilidad como instrumento de garantía para la seguridad alimentaria”. Revista de Derecho civil, vol. III, n. 3, julio-septiembre 2016, pág. 109-138. Disponible en: <http://www.nreg.es/ojs/index.php/RDC/article/view/219/173 > Acceso en: 23 dic. 2020.

MINISTERIO DE AGRICULTURA, PESCA Y ALIMENTACIÓN. Estrategia de digitalización del sector agroalimentario y forestal y del medio natural del Ministerio de Agricultura, Pesca y Alimentación. 2019. Disponible en: < https://www.mapa.gob.es/es/ministerio/planes-estrategias/estrategia-digitalizacion-sectoragroalimentario/estrategia_digitalizacion_sector_agroalimentario_forestal_medio_rural_ve_tcm 30-509645.pdf > Acceso en: 23 dic. 2020.

MINISTERIO DE AGRICULTURA, PESCA Y ALIMENTACIÓN. I Plan de acción 2019-2020 Estrategia de digitalización del sector agroalimentario y forestal y del medio rural. Disponible en: < https://www.mapa.gob.es/es/ministerio/planes-estrategias/estrategiadigitalizacion-sector-agroalimentario/plan-accion-2019-2020-estrategia-digitalizacion_tcm30524904.pdf > Acceso en: 23 dic. 2020.

NOTICIAS PARLAMENTO EUROPEO. "Regulación de la inteligencia artificial en la UE: la propuesta del Parlamento, 2020". 2020. Disponible en: < https://www.europarl.europa.eu/news/es/headlines/society/20201015STO89417/re gulacion-dela-inteligencia-artificial-en-la-ue-la-propuesta-del-parlamento > Acceso en: 23 dic. 2020.

ORGANIZACIÓNDE AVIACIÓNCIVIL INTERNACIONAL. Convenio sobre Aviación Civil Internacional de 7 de diciembre de 1944. Disponible en: $<$ https://www.icao.int/publications/Documents/7300_cons.pdf $\geq$ Acceso en: 22 dic. 2020.

Pantheon. Disponible en: $<$ Pantheon: robots giving a helping hand to hazelnut farmers Shaping Europe's digital future (europa.eu)> Acceso en: 23 dic. 2020.

PINO V., Edwin. "Los drones una herramienta para una agricultura eficiente: un futuro de alta tecnología". Idesia, Vol. 37, núm. 1, 2019, pág. 75-84. Disponible en: $<\underline{\text { https://scielo.conicyt.cl/scielo.php?script=sci_arttext\&pid=S0718-34292019000100075> }}$

Acceso en: 22 de dic. 2020.

RAMÓN FERNÁNDEZ, Francisca. "Robótica, inteligencia artificial y seguridad: ¿Cómo encajar la responsabilidad civil?”. Diario La Ley, n. 9365 2019, pág. 1-13.

RAMÓN FERNÁNDEZ, Francisca. La variedad vegetal ante el avance biotecnológico y los Objetivos de Desarrollo Sostenible, Reus, Madrid, 2020. 

2020.

SmartAgriHubs. 2020. Disponible en: $<$ SmartAgriHubs | Homepage $>$ Acceso en: 23 dic.

Sweeper. Disponible en: <http://www.sweeper-robot.eu/> Acceso en: 22 dic. 2020.

TRIBALDOS CAMPOS, Joaquín. "Drones y nuevas tecnologías para una agricultura inteligente”. Agricultura: Revista agropecuaria y ganadera, n. 1019, 2018, págs. 60-64.

UNIÓN EUROPEA. Comunicación de la Comisión al Parlamento Europeo, al Consejo, al Comité Económico y Social Europeo y al Comité de las Regiones sobre "Una Estrategia para el Mercado Único Digital de Europa (COM/2015/0192 final). 2015. Disponible en: < https://eurlex.europa.eu/legal-content/ES/TXT/?uri=CELEX\%3A52015DC0192 > Acceso en: 23 dic. 2020.

UNIÓN EUROPEA. Reglamento (UE) 2016/679 del Parlamento Europeo y del Consejo, de 27 de abril de 2016, relativo a la protección de las personas físicas en lo que respecta al tratamiento de datos personales y a la libre circulación de estos datos y por el que se deroga la Directiva 95/46/CE (Reglamento general de protección de datos) (Texto pertinente a efectos del EEE). 2016. Disponible en: < https://eur-lex.europa.eu/legalcontent/ES/ALL/?uri=celex\%3A32016R0679> Acceso en: 23 dic. 2020.

UNIÓN EUROPEA. Resolución del Parlamento Europeo, de 7 de junio de 2016, sobre soluciones tecnológicas para una agricultura sostenible en la Unión (2015/2225(INI)). 2016. Disponible en: $\quad<$ https://eur-lex.europa.eu/legalcontent/ES/TXT/?uri=CELEX\%3A52016IP0251\&qid=1608742056010> Acceso en: 23 dic. 2020.

UNIÓN EUROPEA. Resolución del Parlamento Europeo, de 14 de marzo de 2017, sobre las implicaciones de los macrodatos en los derechos fundamentales: privacidad, protección de datos, no discriminación, seguridad y aplicación de la ley (2016/2225(INI)). 2017. Disponible en: $<$ https://op.europa.eu/es/publication-detail/-/publication/bba9b363-8fdf-11e8-8bc101aa75ed71a1 >. Acceso en: 23 dic. 2020.

UNIÓN EUROPEA. Comunicación de la Comisión al Parlamento Europeo, al Consejo, al Comité Económico y social europeo y al Comité de las regiones relativa a la revisión intermedia de la aplicación de la Estrategia para el Mercado Único Digital Un mercado único digital conectado para todos (COM/2017/0228 final). 2017. Disponible en: < https://eurlex.europa.eu/legal-content/ES/TXT/?uri=COM\%3A2017\%3A228\%3AFIN > Acceso en: 23 dic. 2020.

UNIÓN EUROPEA. Reglamento (UE) 2018/1807 del Parlamento Europeo y del Consejo, de 14 de noviembre de 2018, relativo a un marco para la libre circulación de datos no personales en la Unión Europea (Texto pertinente a efectos del EEE). 2018. Disponible en: $<$ https://eurlex.europa.eu/legal-content/es/ALL/?uri=CELEX\%3A32018R1807> Acceso en: 22 dic. 2020.

UNIÓN EUROPEA. Los Estados miembros de la UE unen fuerzas en la digitalización de la agricultura y las zonas rurales europeas, 2019. Disponible en: $<$ https://ec.europa.eu/digitalsingle-market/en/news/eu-member-states-join-forces-digitalisation-european-agriculture-andrural-areas $>$ Acceso en: 22 dic. 2020.

UNIÓN EUROPEA. Libro Blanco sobre la inteligencia artificial-un enfoque europeo orientado a la excelencia y la confianza. 2020. Disponible en: <LIBRO BLANCO sobre la inteligencia artificial - un enfoque europeo orientado a la excelencia y la confianza COM/2020/65 final/2> Acceso en: 22 dic. 2020. 
UNIÓN EUROPEA. Comunicación de la Comisión al Parlamento Europeo, al Consejo, al Comité Económico y social europeo y al Comité de las Regiones sobre una Estrategia Europea de Datos (COM/2020/66 final). 2020. Disponible en: <https:/eur-lex.europa.eu/legalcontent/ES/TXT/?uri=CELEX:52020DC0066> Acceso en: 22 dic. 2020.

UNIÓN EUROPEA. Resolución del Parlamento Europeo, de 20 de octubre de 2020, con recomendaciones destinadas a la Comisión sobre un marco de los aspectos éticos de la inteligencia artificial, la robótica y las tecnologías conexas (2020/2012 (INL)). 2020. Disponible en: https://www.europarl.europa.eu/doceo/document/TA-9-2020-0275_ES.pdf (Consultado el 20 de diciembre de 2020).

UNIÓN EUROPEA. Resolución del Parlamento Europeo, de 20 de octubre de 2020, con recomendaciones destinadas a la Comisión sobre un régimen de responsabilidad civil en materia de inteligencia artificial (2020/2014 (INL)). 2020. Disponible en: < https://www.europarl.europa.eu/doceo/document/TA-9-2020-0276_ES.pdf $>$ Acceso en: 23 dic. 2020.

VÁZQUEZ, A. L., RUIZCANALES, A., MOLINA CABALLERO, J. M. y FERNÁNDEZ PACHECO, D. G.: "Empleo de mini aeronaves no tripuladas (UAV) en gestión de regadíos". Agricultura: Revista agropecuaria, n. 952, 2012, pág. 430-434. Disponible en: < http://www.mapama.gob.es/ministerio/pags/Biblioteca/Revistas/pdf_Agri\%2FAgri_2012_952__ 430_434.pdf > Acceso en: 13 dic. 2017. 